Correspondence

Roxana G. Vitale

rvitale@conicet.gov.ar

Received 13 July 2011

Accepted 8 November 2011

\section{Influence of capsule size on the in vitro activity of antifungal agents against clinical Cryptococcus neoformans var. grubii strains}

\author{
Roxana G. Vitale, ${ }^{1,2}$ Veronica Pascuccelli ${ }^{3}$ and Javier Afeltra ${ }^{2,4}$ \\ ${ }^{1}$ Consejo Nacional de Investigaciones Científicas y Técnológicas (CONICET), Buenos Aires, \\ Argentina \\ ${ }^{2}$ Unidad de Parasitología, Micología, Hospital Ramos Mejía, Buenos Aires, Argentina \\ ${ }^{3}$ División de Análisis Clínicos Sector Biología Molecular, Hospital J.M. Ramos Mejía, Buenos Aires, \\ Argentina \\ ${ }^{4}$ Departamento de Microbiología, Parasitología e Inmunología, Facultad de Medicina, \\ Universidad de Buenos Aires, Buenos Aires, Argentina
}

\begin{abstract}
Cryptococcosis causes disseminated disease in AIDS patients. In contrast to what occurs in laboratory conditions, a large capsule is produced by Cryptococcus neoformans in vivo during infection. The aim of this study was to compare the in vitro activity of different antifungal agents against 34 clinical isolates of $\mathrm{C}$. neoformans var. grubii without or with capsule induction (CLSI, CLSI-C, respectively), following the CLSI M27A3 document. Capsule induction was obtained by addition of $\mathrm{NaHCO}_{3}$ and incubation with $\mathrm{CO}_{2}$. The geometric means of the MICs, in $\mu \mathrm{g} \mathrm{ml}{ }^{-1}$, for CLSI and CLSI-C cultures, respectively, were 1.9 and 9.8 for fluconazole; 0.04 and 0.08 for itraconazole; 0.04 and 0.05 for voriconazole; 0.16 and 0.38 for amphotericin B; and 1.6 and 5.6 for 5-flucytosine. Thus fluconazole showed the highest MICs after capsule induction.

Determination of antifungal activity after capsule induction may be clinically relevant and could be used to evaluate the correlation between in vitro results and clinical outcome.
\end{abstract}

\section{INTRODUCTION}

Cryptococcosis with involvement of the central nervous system is commonly encountered among HIV-infected patients, and approximately $90 \%$ of AIDS patients infected with Cryptococcus neoformans develop meningitis (Nguyen et al., 2010; Osuna et al., 2008). C. neoformans has several virulence factors. Presence of a capsule is the most prominent of these, and it has been shown that capsular polysaccharides have deleterious effects on the immune system (Bahn et al., 2005; Granger et al., 1985).

The size of the capsule of $C$. neoformans is variable, depending on the environmental conditions. Thin capsules are observed in standard fungal culture media in vitro under laboratory conditions. However, during in vivo infection, mainly in cerebrospinal fluid (CSF), the yeasts have large capsules (Frases et al., 2009; Rivera et al., 1998).

Several methodologies to evaluate the antifungal activity of C. neoformans have been proposed by the Clinical and Laboratory Standards Institute (CLSI, formerly NCCLS), in Document M27A3 (CLSI, 2008), but all these procedures

Abbreviations: AMB, amphotericin B; CSF, cerebrospinal fluid; 5FC, flucytosine; FCZ, fluconazole; Gmean, geometric mean; ITZ, itraconazole; VCZ, voriconazole. have to be performed under laboratory conditions, in which $C$. neoformans exhibits a small capsule size.

Some studies have evaluated the antiphagocytic properties of $C$. neoformans after capsule induction by $\mathrm{CO}_{2}$ and sodium bicarbonate (Bahn et al., 2005; Granger et al., 1985; Zaragoza et al., 2003). However, to our knowledge the antifungal activity of different drugs has never been evaluated by the CLSI methodology after capsule induction. Thus, the aim of this work was to study the in vitro activity of different antifungal agents against $C$. neoformans var. grubii in which in vitro capsule growth had been induced to mimic the in vivo situation and to compare these results with those obtained from the same strains without capsule induction.

\section{METHODS}

Antifungal agents. The drugs tested were amphotericin $\mathrm{B}(\mathrm{AMB})$ (Bristol Myers Squibb); fluconazole (FCZ) and voriconazole (VCZ) (Pfizer); itraconazole (ITZ) (Janssen Farmacéutica) and flucytosine (5FC) (Sigma Aldrich).

Organisms and growth conditions. Thirty-four strains of Cryptococcus neoformans isolated from CSF of HIV-positive patients were evaluated. Candida krusei ATCC 6258, Candida parapsilosis 
ATCC 22019, Cryptococcus neoformans ATCC 90112 and C. neoformans acap70 ATCC 52817 (acapsular mutant) were used as qualitycontrol strains. All strains were grown on Sabouraud glucose agar (SGA) at $35^{\circ} \mathrm{C}$ for $48 \mathrm{~h}$ before the susceptibility tests.

Molecular identification. DNA was isolated from the 34 strains following a standard protocol (Liu et al., 2000).

To determine the varietal status of the clinical isolates, the SOD1 allele was amplified by PCR using primers specific for C. neoformans var. grubii and Cryotococcus gattii with the isolated DNA as template. Primers JOHE7777/7775 for C. neoformans var. grubii and JOHE7773/ 7775 for C. gattii were used (D'Souza et al., 2004). Positive controls (C. neoformans var. grubii CBS 8710 and C. gattii WM178) were included.

To determine the serotype and mating type of the clinical isolates, PCR was performed to amplify the mating type $\alpha$ or a and serotype A or D allele of the STE20 locus using specific primers JOHE7270/7272 $(\mathrm{aA})$, JOHE7273/7275 $(\mathrm{aD})$, JOHE7264/7265 $(\alpha \mathrm{A})$ and JOHE7267/ $7268(\alpha \mathrm{D})$ (Barreto de Oliveira et al., 2004). Reference strains CBS $9172(\mathrm{aA})$, CBS $8710(\alpha \mathrm{A})$, CBS 10511(aD) and CBS $10513(\alpha \mathrm{D})$ were included. The mating type $\alpha$ and a primers yielded a $1.2 \mathrm{~kb}$ and an 870 bp product, respectively.

Capsule induction. To induce capsule formation the yeast cells were inoculated on SGA with the addition of $22 \mathrm{mM} \mathrm{NaHCO}_{3}$ and incubated in the presence of $5 \% \mathrm{CO}_{2}$ at $30{ }^{\circ} \mathrm{C}$ for $48 \mathrm{~h}$.

Measurement of capsule. To visualize the size of the capsule a drop of Indian ink was added to a cell suspension. To calculate the size of the capsule the diameter of the cell plus capsule and the cell alone were measured using an optical microscope with a magnification of $\times 400$ using a calibrated micrometer eyepiece. Capsule size was obtained by the difference between the size of the cell plus capsule, and the size of the yeast cell alone. Thirty measurements were made for each determination, and the mean was calculated.

Antifungal susceptibility testing. The MICs were determined following the CLSI M27A3 guidelines (CLSI, 2008) in two conditions: (a) without capsule induction (CLSI) and (b) with capsule induction (CLSI-C). For (a), the isolates were incubated for $48 \mathrm{~h}$ on SGA before the tests. The medium used for the tests was RPMI 1640 without sodium bicarbonate, with L-glutamine (Sigma Aldrich), buffered with MOPS (Sigma Aldrich) at $\mathrm{pH}$ 7. For (b), after capsule induction as described above, the isolates were grown in RPMI 1640 medium containing $22 \mathrm{mM} \mathrm{NaHCO}_{3}$, with L-glutamine, buffered with MOPS at $\mathrm{pH}$ 7, and the incubation was performed in the presence of $5 \% \mathrm{CO}_{2}$ to allow capsule induction. The inoculum was adjusted by haemocytometer measurements. The starting inoculum was $0.5-2.5 \times 10^{6}$ c.f.u. $\mathrm{ml}^{-1}$, and the final inoculum was $0.5-2.5 \times 10^{3}$ c.f.u. $\mathrm{ml}^{-1}$, for both (a) and (b).

The inoculum size was checked by plating serial dilutions on SGA and c.f.u. were determined after incubation at $35{ }^{\circ} \mathrm{C}$ for up to $72 \mathrm{~h}$.

AMB, ITZ and VCZ were diluted in DMSO at $3200 \mu \mathrm{g} \mathrm{ml}^{-1}$, whereas FCZ and 5FC were diluted in water at $12800 \mu \mathrm{g} \mathrm{ml}^{-1}$. Serial dilutions of the drugs were made following the CLSI guidelines in order to obtain final concentration ranges of $16-0.03 \mu \mathrm{g} \mathrm{ml}^{-1}$ for AMB; $8-0.007 \mu \mathrm{g}$ $\mathrm{ml}^{-1}$ for ITZ and VCZ, 64-0.06 for FCZ and 32-0.03 for 5FC. The tests were performed in 96-well flat-bottomed microtitre plates (Becton Dickinson). Yeast suspensions were diluted $1: 10$ and $1: 100$ in water and in liquid RPMI 1640 medium (see above). The plates were incubated at $35{ }^{\circ} \mathrm{C}$ for $72 \mathrm{~h}$, then scanned at $450 \mathrm{~nm}$ with a Labsystems Multiskan RC microplate spectrophotometer (Labsystems Multiskan).

The MIC end point for AMB was defined as the lowest concentration of the drug that showed $100 \%$ inhibition compared with the growth control; for the other drugs it was defined as the lowest concentration that showed $50 \%$ of inhibition compared with the control. All tests were performed in duplicate on two different days.

Statistical analysis. The high and the low off-scale MICs were included in the analysis. High off-scale MICs were converted to the next higher concentration and the low off-scale MICs were converted to the next lower concentration. The differences between MICs obtained by the different methods were analysed by Mann-Whitney $U$ test. The significance level of 0.05 was chosen.

\section{RESULTS}

\section{In vitro capsule induction}

Capsule size was measured after incubation on SGA with or without induction by $\mathrm{NaHCO}_{3}$ in the presence of $5 \% \mathrm{CO}_{2}$. For the C. neoformans acapsular mutant ATCC 52817, no modification was observed under these conditions. For the $C$. neoformans clinical strains and the reference strain ATCC 90112, capsule size was significantly increased from 1-3 $\mu \mathrm{m}$ to $10-15 \mu \mathrm{m}$ after induction.

The geometric mean (Gmean) in $\mu \mathrm{m}$, for capsule size without and with in vitro induction for acapsular C. neoformans ATCC 52817 was 1 for both conditions; for the clinical isolates of C. neoformans var. grubii $(n=34)$ it was 2.6 and 12.1 , respectively.

The MICs for the control strains Candida krusei ATCC 6258 and Candida parapsilosis ATCC 22019 were within the expected range proposed by the CLSI M27A3 document.

\section{Molecular analysis of clinical isolates}

The PCR with SOD1-C. gattii-specific primers yielded products with the reference $C$. gattii strains, but no product with the clinical isolates was detected, indicating that none of these strains were C. gattii. The C. neoformans var. grubiispecific primers gave PCR products with all the samples tested, indicating that all of them were C. neoformans var. grubii. PCR amplification using primers specific for the STE20 gene showed that all the isolates were of serotype A. For the mating type test, the $\alpha \mathrm{A}$ PCR gave positive results with all the strains except one, which was an aA isolate.

\section{Antifungal susceptibility testing}

In general, higher MIC values were observed for encapsulated strains. FCZ was the least active drug: the Gmean MICs ( $\mu$ g $\mathrm{ml}^{-1}$ ) for CLSI and CLSI-C were 1.9 and $9.8(P<0.05)$. Fourteen strains with small capsules for which the MIC values were low showed an increase in MIC of more than two dilution steps when tested with a large capsule (Fig. 1a). 5FC and $\mathrm{AMB}$ also showed a significant difference in MIC between the two methodologies, the respective Gmean values being 1.6 and $5.6 \mu \mathrm{g} \mathrm{ml}^{-1}$ for 5FC (Fig. 1e), and 0.16 and $0.38 \mu \mathrm{g} \mathrm{ml}^{-1}$ for AMB (Fig. 1d), for CLSI and CLSI-C, respectively $(P<0.05)$. However, for 5FC only in eight strains was a difference of more than two dilution steps observed. For the other drugs no statistically significant difference was 

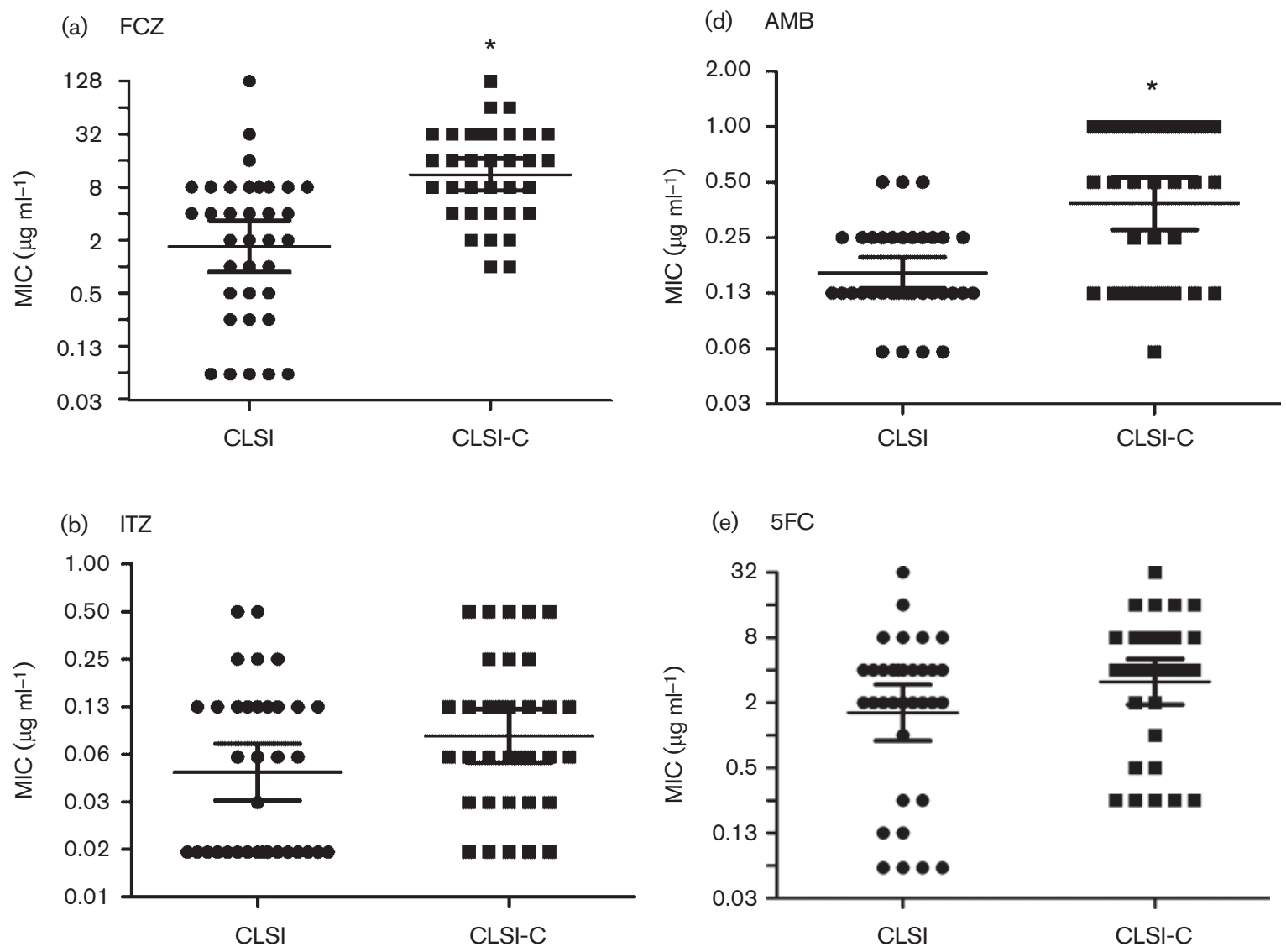

(e) $5 \mathrm{FC}$

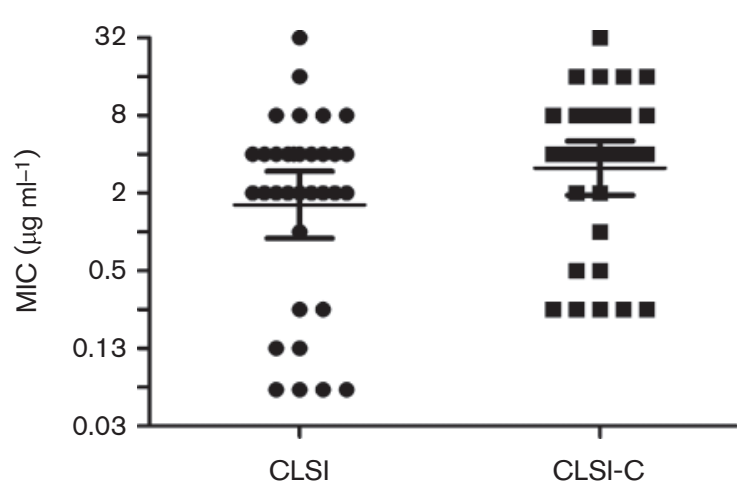

(c) $\mathrm{VCZ}$

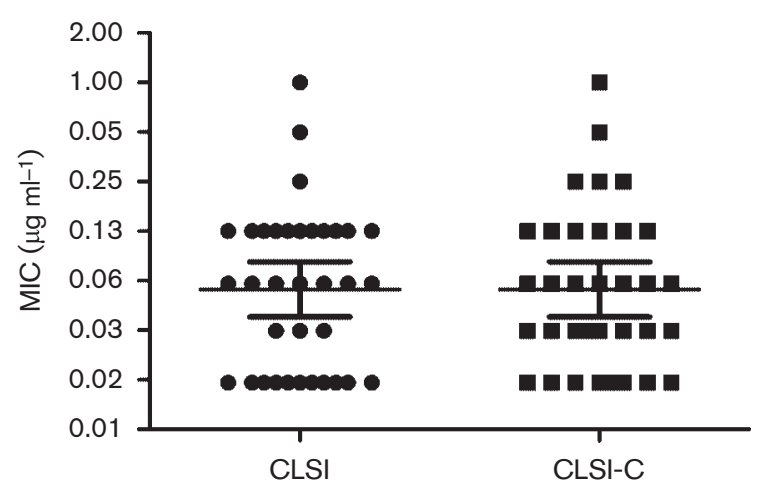

Fig. 1. Comparison of the MICs $\left(\mu \mathrm{g} \mathrm{ml}^{-1}\right)$ between CLSI and CLSI-C methodology for the antifungal agents tested. ${ }^{*}, P<0.05$.

observed $(P>0.05)$ : the respective Gmean MICs in $\mu \mathrm{g} \mathrm{ml}^{-1}$ determined by CLSI and CLSI-C, respectively, were 0.04 and 0.08 for ITZ (Fig. 1b), and 0.04 and 0.05 for VCZ (Fig. 1c). All data are summarized in Table 1.

\section{DISCUSSION}

Cryptococcus neoformans has a large polysaccharide capsule that is necessary for virulence and is the target of a protective antibody response (Frases et al., 2009; Zaragoza et al., 2009). Variation of capsule size between different cryptococcal isolates during the course of human infection has been reported (Cherniak et al., 1995), and capsule size may also vary depending on the site of infection (Rivera et al., 1998). The mechanisms responsible for changes in the thickness of the capsule could be related to different proteins involved in its biosynthesis (Janbon et al., 2001). The gas carbon dioxide $\left(\mathrm{CO}_{2}\right)$ plays a critical role in microbial and mammalian respiration and it is known to 
Table 1. Gmean MICs, $\mathrm{MIC}_{50}, \mathrm{MIC}_{90}$ and $\mathrm{MIC}$ range of the drugs tested against $C$. neoformans var. grubii

All values are in $\mu \mathrm{g} \mathrm{ml}{ }^{-1}$.

\begin{tabular}{|c|c|c|c|c|c|c|c|c|}
\hline \multirow[t]{2}{*}{ Drug } & \multicolumn{4}{|c|}{ (a) CLSI } & \multicolumn{4}{|c|}{ (b) CLSI-C } \\
\hline & Gmean & $\mathrm{MIC}_{50}$ & $\mathrm{MIC}_{90}$ & Range & Gmean & $\mathrm{MIC}_{50}$ & $\mathrm{MIC}_{90}$ & Range \\
\hline ITZ & 0.04 & 0.06 & 0.25 & $0.015-0.5$ & 0.08 & 0.06 & 0.5 & $0.015-0.5$ \\
\hline VCZ & 0.04 & 0.06 & 0.125 & $0.015-0.5$ & 0.05 & 0.06 & 0.25 & $0.015-1$ \\
\hline FCZ & 1.9 & 4 & 8 & $0.06-128$ & 9.8 & 16 & 32 & $0.5-128$ \\
\hline
\end{tabular}

induce production of polysaccharide capsule in pathogenic bacteria and fungi (Bahn et al., 2005). However, the mechanism by which this occurs has not yet been clarified. In human infection, larger $C$. neoformans capsule sizes were especially observed in CSF (Bose et al., 2003).

The role of the capsule in the antifungal susceptibility of C. neoformans is poorly known. Therefore, we studied antifungal activity against isolates of $C$. neoformans var. grubii, identified by classical and molecular techniques, in which capsule production was induced in vitro by incubation with bicarbonate and $\mathrm{CO}_{2}$. The results were compared with those obtained for the same strains by cultivation under standard laboratory conditions. There is no standard definition of 'small' and 'large' capsules. In one study of proven pulmonary cryptococcosis, C. neoformans cells with a capsule size $\leqslant 1 \mu \mathrm{m}$ were called capsule deficient, whereas those with a capsule size $>1 \mu \mathrm{m}$ were defined as having a large capsule (Torres et al., 2005). In the present study, we defined small capsulated strains as those with capsule sizes $1-3 \mu \mathrm{m}$ and large capsulated strains as those with capsule sizes $>3 \mu \mathrm{m}$.

All the strains tested when incubated in the CLSI standard conditions showed a capsule size of 1-2 $\mu \mathrm{m}$, whereas with the addition of $\mathrm{NaHCO}_{3}$ and in the presence of $\mathrm{CO}_{2}$, the size increased up to $15 \mu \mathrm{m}$, depending on the strain. In contrast, no induction was observed for the control strain, the acapsulate mutant C. neoformans ATCC 52817.

The presence of bicarbonate and $\mathrm{CO}_{2}$ did not affect the growth of the Candida control strains or the C. neoformans isolates, which is in agreement with another study in which hypoxic conditions and presence of $\mathrm{CO}_{2}$ did not influence growth of Candida and Aspergillus (Warn et al., 2004). On the other hand, the antifungal MICs for the Candida control strains were similar in the presence or absence of bicarbonate and $\mathrm{CO}_{2}$, indicating they did not influence the MIC determination.

The MICs observed in this study differed significantly among the drugs and the methodologies. Capsule size is altered by VCZ (van Duin et al., 2004) and this effect could be related to the fact that no differences were observed in the MIC of this drug between small and large capsulated strains.
FCZ was the least active drug in the presence of a large capsule. The reason why capsulated strains are less susceptible to this azole cannot be explained by the results of the present study. It is possible that capsule components reduce the ability of this drug to attach to the target, resulting in the lack of action observed. However, for the other azoles this effect was not observed. It may be necessary to study this phenomenon at molecular level in order to elucidate if the mechanism of action in encapsulated $C$. neoformans strains varies among the azoles. The finding that FCZ is less active is remarkable, since this drug is used in treatment of cryptoccocal meningitis after AMB therapy.

For 5FC, significant differences in antifungal activity were observed between large and small encapsulated strains, but the difference was no more than two dilution steps for most of the strains. The MIC values observed in this work are similar to those reported by other authors that evaluated the activity of 5FC against $C$. neoformans without capsule induction (Chowdhary et al., 2011; Dannaoui et al., 2006). It seems that the polysaccharide capsule may affect the activity of 5FC differently from the other drugs; this could be related to the small size of this drug and its mechanism of action.

AMB was significantly less active for large capsulated strains. In one study it was demonstrated that AMB may affect the capsule size in a murine model (Zaragoza et al., 2005). In our in vitro study, however, it seems that capsule could affect the activity of AMB. These are interesting observations, despite the fact that the two studies differed in the methodology used. Since when present in the CSF C. neoformans is usually seen with a large capsule, our observation of the effect of encapsulation on the activity of this polyene could be one of the reasons why in several clinical trials of $\mathrm{AMB}$ alone, it showed little ability to remove the fungus from the CSF in patients with disseminated cryptococcosis with CNS involvement (Perfect et al., 2010).

In conclusion, this work has demonstrated that the presence of a polysaccharide capsule can influence the susceptibility of $C$. neoformans var. grubii to some antifungal agents. In vitro susceptibility tests of $C$. neoformans are routinely performed using strains with a thin capsule, 
which is how $C$. neoformans appears outside the human body. Because during human infection, yeast cells with large capsules are generally present, performing MIC determinations after capsule induction could be a promising tool. It will be necessary to evaluate the optimal conditions to analyse the in vitro susceptibility of $C$. neoformans in order to find better approaches to correlate the results of in vitro tests with the clinical outcome. Thus, further studies are needed in order to elucidate the usefulness of these findings.

\section{ACKNOWLEDGEMENTS}

The authors acknowledge Eric Dannaoui for helpful discussions on the paper, Ferry Hagen for providing the C. neoformans variety grubii and C. gattii reference strains and Laura Rodero for providing several of the tested strains.

\section{REFERENCES}

Bahn, Y. S., Cox, G. M., Perfect, J. R. \& Heitman, J. (2005). Carbonic anhydrase and $\mathrm{CO}_{2}$ sensing during Cryptococcus neoformans growth, differentiation, and virulence. Curr Biol 15, 2013-2020.

Barreto de Oliveira, M. T., Boekhout, T., Theelen, B., Hagen, F., Baroni, F. A., Lazera, M. S., Lengeler, K. B., Heitman, J., Rivera, I. N. \& Paula, C. R. (2004). Cryptococcus neoformans shows a remarkable genotypic diversity in Brazil. J Clin Microbiol 42, 1356-1359.

Bose, I., Reese, A. J., Ory, J. J., Janbon, G. \& Doering, T. L. (2003). A yeast under cover: the capsule of Cryptococcus neoformans. Eukaryot Cell 2, 655-663.

Cherniak, R., Morris, L. C., Belay, T., Spitzer, E. D. \& Casadevall, A. (1995). Variation in the structure of glucuronoxylomannan in isolates from patients with recurrent cryptococcal meningitis. Infect Immun 63, 1899-1905.

Chowdhary, A., Randhawa, H. S., Sundar, G., Kathuria, S., Prakash, A., Khan, Z., Sun, S. \& Xu, J. (2011). In vitro antifungal susceptibility profiles and genotypes of 308 clinical and environmental isolates of Cryptococcus neoformans var. grubii and Cryptococcus gattii serotype B from north-western India. J Med Microbiol 60, 961-967.

CLSI (2008). Reference Method for Broth Dilution Antifungal Susceptibility Testing of Yeasts. Approved Standard. Document M27A3. Wayne, PA: Clinical Laboratory Standards Institute.

D'Souza, C. A., Hagen, F., Boekhout, T., Cox, G. M. \& Heitman, J. (2004). Investigation of the basis of virulence in serotype A strains of Cryptococcus neoformans from apparently immunocompetent individuals. Curr Genet 46, 92-102.

Dannaoui, E., Abdul, M., Arpin, M., Michel-Nguyen, A., Piens, M. A., Favel, A., Lortholary, O., Dromer, F. \& French Cryptococcosis Study Group (2006). Results obtained with various antifungal susceptibility testing methods do not predict early clinical outcome in patients with cryptococcosis. Antimicrob Agents Chemother 50, 2464-2470.

Frases, S., Pontes, B., Nimrichter, L., Rodrigues, M. L., Viana, N. B. \& Casadevall, A. (2009). The elastic properties of the Cryptococcus neoformans capsule. Biophys J 97, 937-945.

Granger, D. L., Perfect, J. R. \& Durack, D. T. (1985). Virulence of Cryptococcus neoformans. Regulation of capsule synthesis by carbon dioxide. J Clin Invest 76, 508-516.

Janbon, G., Himmelreich, U., Moyrand, F., Improvisi, L. \& Dromer, F. (2001). Caslp is a membrane protein necessary for the $O$-acetylation of the Cryptococcus neoformans capsular polysaccharide. Mol Microbiol 42, 453-467.

Liu, D., Coloe, S., Baird, R. \& Pederson, J. (2000). Rapid minipreparation of fungal DNA for PCR. J Clin Microbiol 38, 471.

Nguyen, M. H., Husain, S., Clancy, C. J., Peacock, J. E., Hung, C. C., Kontoyiannis, D. P., Morris, A. J., Heath, C. H., Wagener, M. \& Yu, V. L. (2010). Outcomes of central nervous system cryptococcosis vary with host immune function: results from a multi-center, prospective study. J Infect 61, 419-426.

Osuna, A., Carragoso, A., Lemos, A., Mocho, M. L. \& Gaspar, O. (2008). [Cryptococcosis]. Acta Med Port 21, 307-313 (in Portuguese).

Perfect, J. R., Dismukes, W. E., Dromer, F., Goldman, D. L., Graybill, J. R., Hamill, R. J., Harrison, T. S., Larsen, R. A., Lortholary, O. \& other authors (2010). Clinical practice guidelines for the management of cryptococcal disease: 2010 update by the Infectious Diseases Society of America. Clin Infect Dis 50, 291-322.

Rivera, J., Feldmesser, M., Cammer, M. \& Casadevall, A. (1998). Organ-dependent variation of capsule thickness in Cryptococcus neoformans during experimental murine infection. Infect Immun 66, 5027-5030.

Torres, H. A., Prieto, V. G., Raad, I. I. \& Kontoyiannis, D. P. (2005). Proven pulmonary cryptococcosis due to capsule-deficient Cryptococcus neoformans does not differ clinically from proven pulmonary cryptococcosis due to capsule-intact Cr. neoformans. Mycoses 48, 21-24.

van Duin, D., Cleare, W., Zaragoza, O., Casadevall, A. \& Nosanchuk, J. D. (2004). Effects of voriconazole on Cryptococcus neoformans. Antimicrob Agents Chemother 48, 2014-2020.

Warn, P. A., Sharp, A., Guinea, J. \& Denning, D. W. (2004). Effect of hypoxic conditions on in vitro susceptibility testing of amphotericin $\mathrm{B}$, itraconazole and micafungin against Aspergillus and Candida. J Antimicrob Chemother 53, 743-749.

Zaragoza, O., Fries, B. C. \& Casadevall, A. (2003). Induction of capsule growth in Cryptococcus neoformans by mammalian serum and $\mathrm{CO}_{2}$. Infect Immun 71, 6155-6164.

Zaragoza, O., Mihu, C., Casadevall, A. \& Nosanchuk, J. D. (2005). Effect of amphotericin B on capsule and cell size in Cryptococcus neoformans during murine infection. Antimicrob Agents Chemother 49, 4358-4361.

Zaragoza, O., Rodrigues, M. L., De Jesus, M., Frases, S., Dadachova, E. \& Casadevall, A. (2009). The capsule of the fungal pathogen Cryptococcus neoformans. Adv Appl Microbiol 68, 133-216. 